\title{
Topological and Central Trigger Processor for 2014 LHC luminosities
}

G. Anders ${ }^{b, c}$, B. Bauss ${ }^{a}$, D. Berge ${ }^{c}$ V. Büscher ${ }^{a}$, T. Childers ${ }^{c}$, R. Degele $^{a}$, E. Dobson ${ }^{c}$, A. Ebling ${ }^{a}$, N. Ellis ${ }^{c}$ P. Farthouat ${ }^{c}$ C.

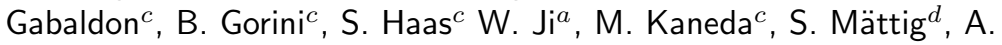

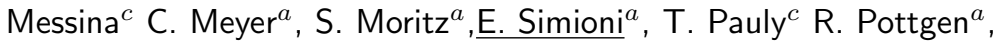
U. Schäfer ${ }^{a}$, R. Spiwoks ${ }^{c}$ S. Tapprogge ${ }^{a}$, T. Wengler $^{c}$, V. Wenzel ${ }^{a}$

a, Institut für Physik, Universität Mainz, Mainz, Germany $b$, Kirchhoff-Institut für Physik, Ruprecht-Karls-Universität Heidelberg, Heidelberg, Germany c, CERN, Geneva, Switzerland d, DESY, Hamburg and Zeuthen, Germany 


\section{Overview}

(1) ATLAS Level-1 Trigger 2013-2014 Upgrade

2 Central trigger processor upgrade

3 Topological Processor

(4) Topological Processor functional demonstrator test results

(5) Summary 


\section{ATLAS Detector}

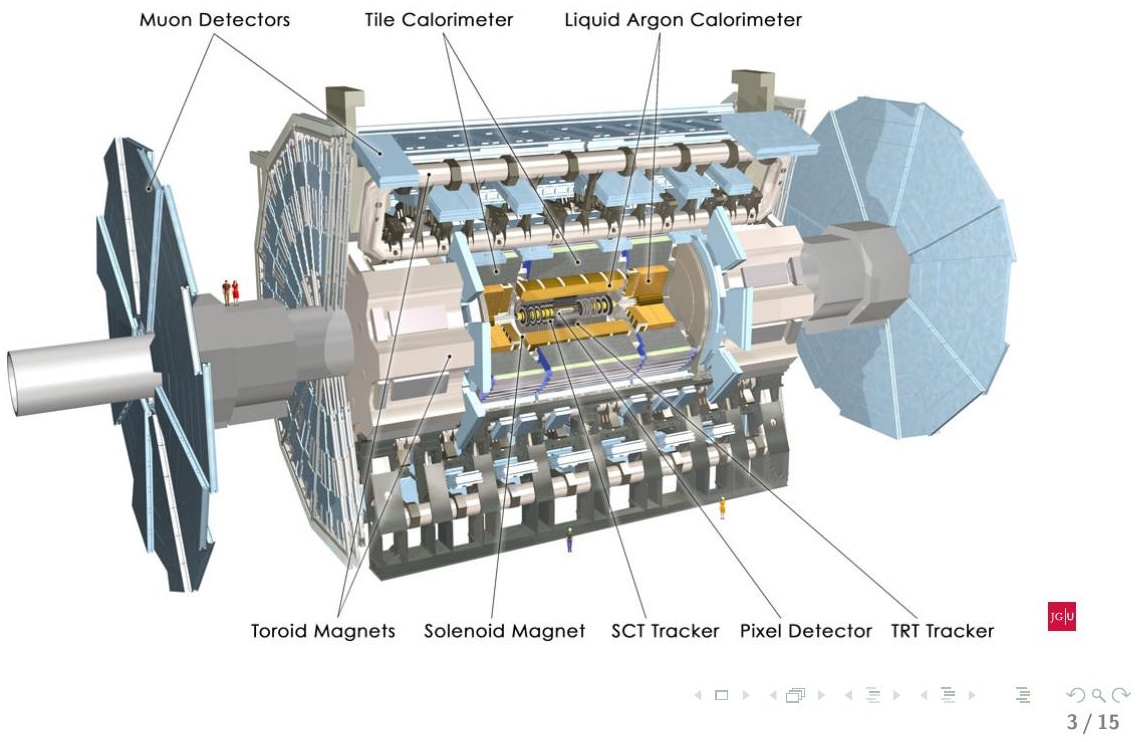




\section{ATLAS Level-1 Trigger}

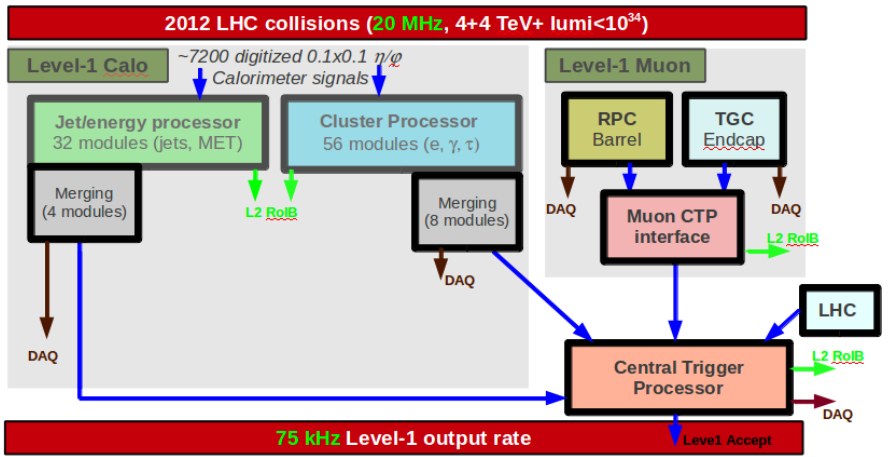

- Hardware based, synchronous with LHC clock

- Pipelines $2.5 \mu \mathrm{s}$ fixed latency system

- The output is a single bit "Level-1 Accept" (L1A) signaling to the detector front-end readout systems via the Trigger Timing and Control system (TTC) 


\section{Level-1 upgrade in 2013-2014 (Phase-0 upgrade)}

Motivation:

- LHC proton collisions at $14 \mathrm{TeV}$ and luminosity above $3 \times 10^{34} \mathrm{~cm}^{-2} \mathrm{~s}^{-1}$

- Higher events rate

Strategy for the Level-1 trigger upgrade:

- Use geometrical information of jets/leptons/MET and apply topology cuts (currently used @ Level-2)

- Replace the Common Merging Modules (optical connectivity)

- Add a new hardware element: Topological Processor

- Upgrade the Central Trigger Processor (optical connectivity and more resources)

See also the talk of Riccardo Vari, UPG2-2, "Upgrade project and plans for the ATLAS detector and trigger" 


\section{CTP Resource Utilisation}

\begin{tabular}{|l|r|r|}
\hline What & Used & 9 \\
\hline CTPIN input cables (partially used) & 212 & 12 \\
\hline CTPIN input signals* & 160 & 372 \\
\hline CTP PIT bus lines & 1 & 160 \\
\hline CTPCAL calibration request ** & 241 & 19 \\
\hline CTPCORE trigger items & 8 & 256 \\
\hline CTPCORE bunch groups & 6 & 8 \\
\hline CTPCORE maximum number of AND terms *** & 6 & 256 \\
\hline CTPCORE maximum number of bits in OR terms & 20 & 12 \\
\hline CTPOUT output cables to TTC partitions & 138 & 20 \\
\hline CTPIN integrating monitoring counters & 88 & 768 \\
\hline CTPMON per-bunch monitoring counters & 12 & 160 \\
\hline CTPCORE per-bunch trigger item counters & 12 \\
\hline
\end{tabular}

* = dedicated patch of CTPIN firmware to reduce 16 MBTS signals to 3-bit value, and some input signals are only used for CTPIN monitoring

** = only TILE sub-detector

$* * *=$ always two terms are used for bunch groups 


\section{ATLAS Phase-0 upgraded Level-1 Trigger}

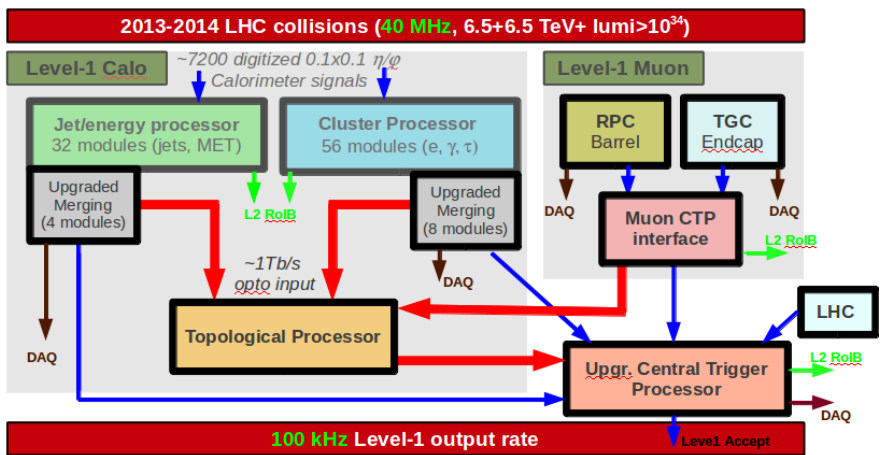

The main requirement is to provide optical connectivity for the TP to apply topological cuts, double the CTP trigger input (and trigger items) and add secondary trigger partitions. 


\section{Phase-0 upgrade CTP features}

- Current CTP functionalities

- Receives and aligns trigger inputs and produces trigger menu items

- Forms the Level-1 decision and generates dead-time (L1A generation)

- Monitors input trigger rates and applies pre-scaling and veto

- Receives timing signals (BC, ORBIT) from the LHC

- Sends Rol information to LVL2, and read-out data to DAQ

- Distributes timing signals and trigger decision to all sub-detectors

- CTP new features

- Increase resources (trigger inputs and trigger items)

- Optical inputs to connect to Topo Processor (and MUCTPI)

- Improved bunch group masking/monitoring (i.e. "collision bunch group" contains the BCIDs of collidingbunches)

- Allow partitioning of Level-1 Accept generation for detector commissioning

- CTP upgrade requires complete redesign of several modules 


\section{Phase-0 upgrade CTP Specification}

- 320 trigger inputs on PIT bus backplane (now 160)

- 512 trigger items (now 256)

- 12 optical inputs using ribbon-fiber receiver

- Avago MiniPOD for compatibility with Topo Processor

- 16 bunch groups (now 8)

- 256 per-bunch counters for trigger item monitoring (now 12)

- 4 trigger partitions

- Common trigger menu

- Each L1A partition has selection of trigger items, and it's own deadtime handling

- Only primary L1A partition (i.e. "physics partition") provides LVL2 and DAQ readout

- Secondary L1A partitions used for detector commissioning, calibration, etc. 


\section{Phase-0 Topological Processor}

- The Topological Processor is a new element in the trigger

- Allow for any conceivable topological algorithm

- Route a maximum fibre count / data volume into individual module / processor

- Electrons, tau, jets, MET : $553 \mathrm{~Gb} / \mathrm{s}$

- Muons $267 \mathrm{~Gb} / \mathrm{s}$

- Total aggregate bandwidth $820 \mathrm{~Gb} / \mathrm{s}$

- Achievable at $6.4 \mathrm{~Gb} / \mathrm{s}$ line rate

- Design

- Fully ATCA compliant

- Optical input from the backplane via blindmate connectors

- High-density midboard o/e converters (AVAGO miniPOD)

- Keep compact, with short traces and no on-board signal duplication

- FPGA based processors (Xilinx Virtex7 XC7V485T initially)

- Output from opto links on the front panel to the CTP 


\section{CTPCORE ++ and TP modules}

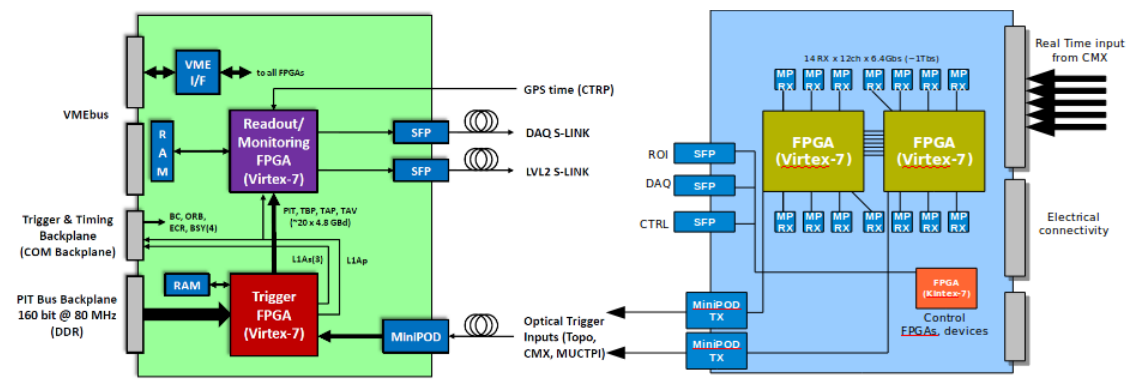




\section{TP Functional Demonstrator "GOLD"}

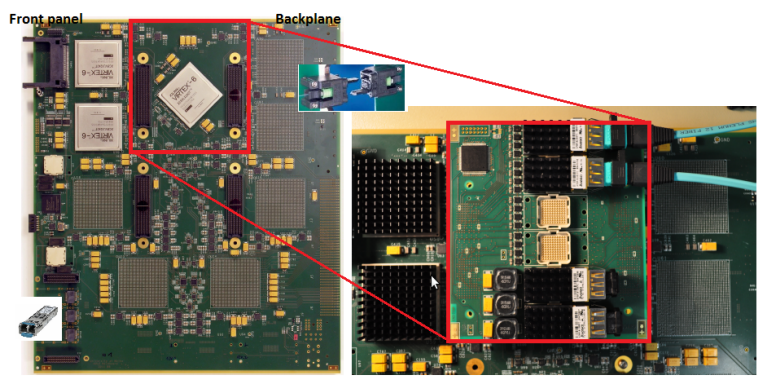

- ATCA form factor

- Input from the backplane to opto-mezzanine

- $10 \mathrm{~Gb} / \mathrm{s}$ (up to) AVAGO o/e converters

- High performing FPGAs (XC6VLX240T) with multiple 6.4 $\mathrm{Gb} / \mathrm{s}$ transceivers

- Output from opto links on the front panel

- An useful "gym" for testing technology needed for the TP 


\section{Tests performed on "GOLD"}
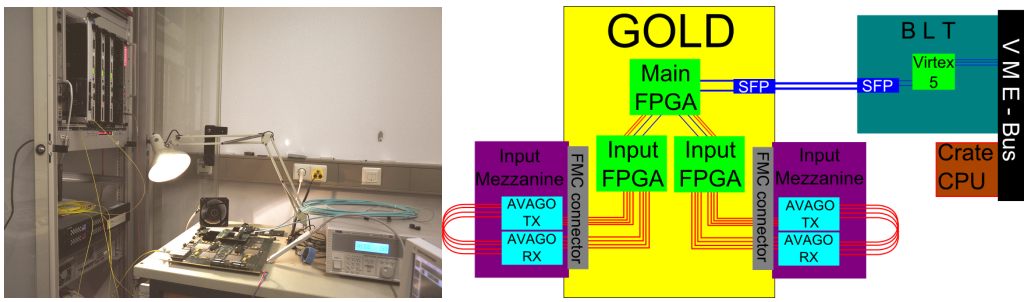

- Initial verification of clocks and boundary scans

- Full data path betwen FPGAs - o/e converter loopback and Bit Error Rate scan

- Check of signal integrity ("Eye diagrams")

- Measured error free time regions ("Bathtub profiles")

- Achieve optimization of the transceivers parameters

- Latency measurements

- Logic utilization by algorithms 


\section{Test results of the full data path at $6.4 \mathrm{~Gb} / \mathrm{s}$}
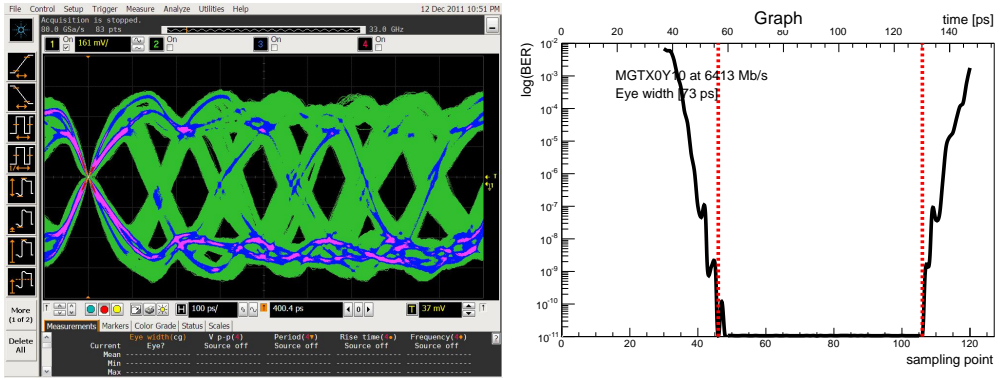

- Full real time data path working at required speed 


\section{Summary}

- Central Trigger Processor Phase-0 upgrade

- Double the number of trigger inputs $(160 \rightarrow 320)$

- Double the number of trigger items $(256 \rightarrow 512)$

- Secondary trigger partitions for detector commissioning

- Design review Sep/Oct 2012

- First prototype in Q1 2013

- Aim to be ready in 2014 ATLAS combined cosmics running

- Topological Processor

- Allow to apply topological cuts at Level-1

- Large physics potential and many simulation studies carried out

- Design review today

- First prototype in Q2 2013 (well in line with CTP)

- Large campaign of tests successfull performed on the TP functional demonstrator "GOLD"

- Aim to be ready in 2014 ATLAS combined cosmics running 